\title{
Muséologies
}

Les cahiers d'études supérieures

muséologies

\section{Collections universitaires dormantes : bilan et perspective}

\section{Lydia Bhérer-Vidal}

Volume 2, numéro 1, octobre 2007

URI : https://id.erudit.org/iderudit/1033601ar

DOI : https://doi.org/10.7202/1033601ar

Aller au sommaire du numéro

Éditeur(s)

Association Québécoise de Promotion des Recherches Étudiantes en Muséologie (AQPREM)

ISSN

1718-5181 (imprimé)

1929-7815 (numérique)

Découvrir la revue

Citer cet article

Bhérer-Vidal, L. (2007). Collections universitaires dormantes : bilan et perspective. Muséologies, 2(1), 120-136. https://doi.org/10.7202/1033601ar
Résumé de l'article

Les collections universitaires sont riches et variées. Pourtant, de par leur caractère spécialisé, mais aussi suite à l'évolution des technologies et des nouvelles méthodes pédagogiques, elles sont de nos jours, rarement utilisées pour l'enseignement. Ce changement de vocation explique l'état actuel de dormance de ces collections universitaires. L'auteur s'attarde sur ces changements et questionne l'avenir de ce patrimoine, en proposant des solutions pour favoriser leur emploi. 
Collection universitaires dormantes :

bilan et perspective

1

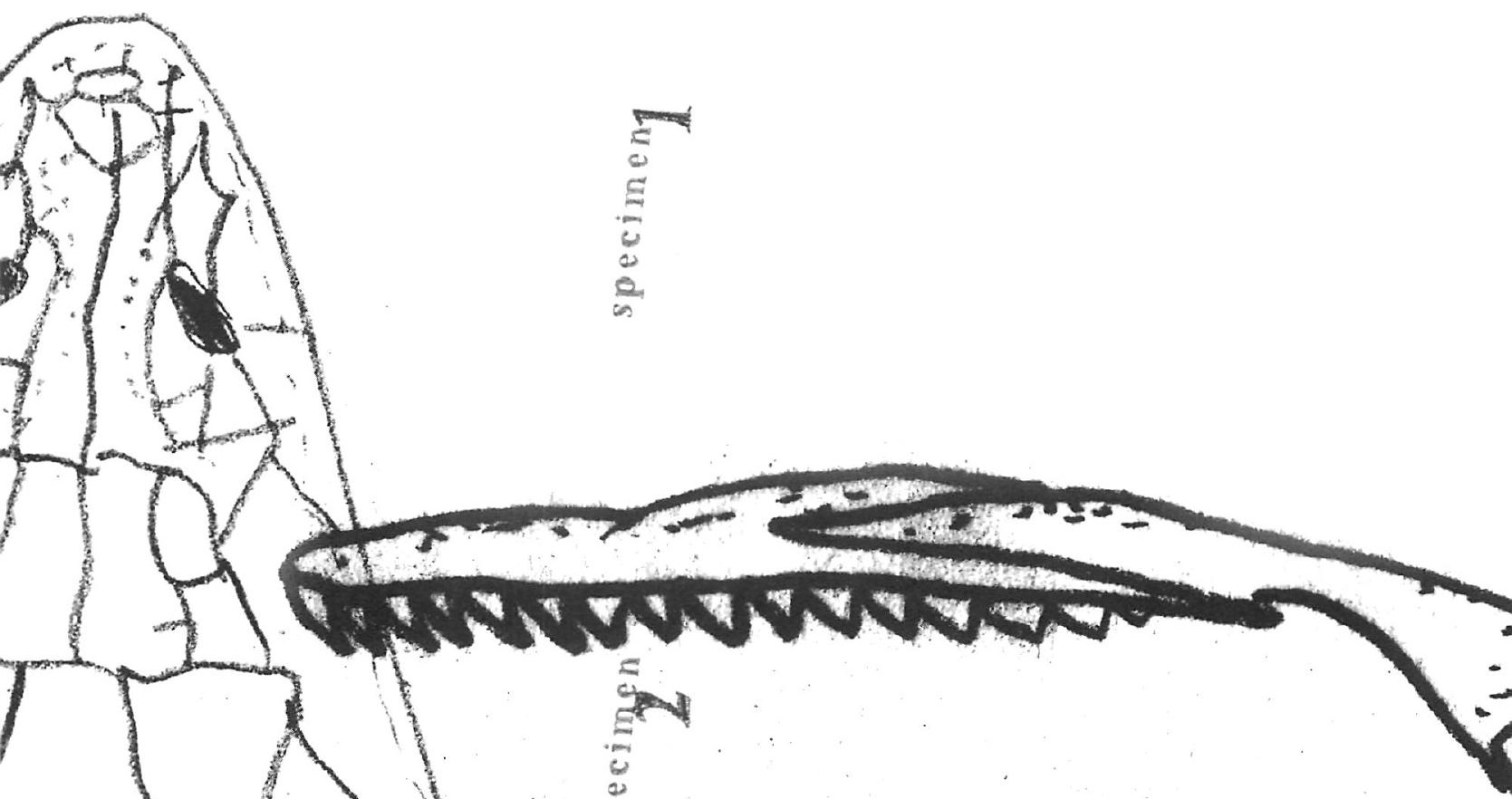


Les collections universitaires sont riches et variées. Pourtant, de par leur caractère spécialisé, mais aussi suite à l'évolution des technologies et des nouvelles méthodes pédagogiques, elles sont de nos jours, rarement utilisées pour l'enseignement. Ce changement de vocation explique l'état actuel de dormance de ces collections universitaires. L'auteur s'attarde sur ces changements et questionne l'avenir de ce patrimoine, en proposant des solutions pour favoriser leur emploi.

LYDIA BHÉRER-VIDAL DÉTIENT UN DIPLÔME DE PREMIER CYCLE EN HISTOIRE DE LART ET ARCHÉOLOGIE DE L'UNIVERSITÉ PAUL-VALÉRY (MONTPELLIER, FRANCE) ET UN BACCALAURÉAT EN HISTOIRE DE L'ART DE L'UNIVERSITÉ LAVAL. ELLE A NOTAMMENT PARTICIPÉ À L'ORGANISATION DE DEUX EXPOSITIONS D'ART CONTEMPORAIN ET D'UN COLLOQUE DE MUSÉOLOGIE ET A TRAVAILLÉ À L'INVENTAIRE DE L'ART PUBLIC POUR LE MINISTÈRE DE LA CULTURE ET DES COMMUNICATIONS DU QUÉBEC. ELLE A PAR LA SUITE OBTENU SON DESS (DIPLÔME D'ÉTUDES SUPÉRIEURES SPÉCIALISÉES) EN MUSÉOLOGIE À L'UNIVERSITÉ LAVAL EN 2007, PUIS A AGI COMME CHARGÉE DE CONSERVATION DU FONDS DOCUMENTAIREDULABORATOIREDEMUSÉOLOGIEET D'INGÉNIERIEDE LA CULTURE (LAMIC) À CETTE MÊME UNIVERSITÉ. 
LOURENÇO, Marta C.

«Musées et collections des universités : les origines". La Revue, $\mathrm{n}^{\circ} 41$, mai 2004 ,

p. 51-61

[2]

LOURENÇO, Marta C. Entre deux mondes : La spécificité et le rôle contemporain des collections et musées des universités en Europe. Thèse de doctorat en muséologie, sous la direction de Dominique Ferriot et Steven de Clercq. Conservatoire national des arts et métiers, Paris, 2005, p. 81.
Le premier musée, dans l'acception moderne du terme, à avoir vu le jour est un musée universitaire : l'Ashmolean Museum d'Oxford, en Angleterre, inauguré en 1683 ; il s'agissait d'une institution permanente, possédant des collections et ouverte au public.

Cependant, l'Ashmolean Museum ne s'est pas réalisé en un jour. Bien avant le XVII siècle, les universités européennes possédaient des collections qu'elles pouvaient présenter à un public plus large que celui des étudiants et des professeurs.

Selon les spécialistes de la question, quatre étapes ont mené à "l'invention du musée universitaire " 11 : la constitution de collections d'enseignement lors de la période médiévale; la mise en place, en Italie, du jardin médicinal (hortus medicus) et du théâtre anatomique (theatrum anatomicum) dans la deuxième moitié du XVI siècle; la création de "musées d'enseignement " au XVII' siècle et la création des collections d'étude, du XVI ${ }^{e}$ au XVIII siècle.

L'ouverture, en mai 1683, de l'Ashmolean Museum à Oxford, l'exemple même du musée universitaire moderne, marque réellement «l'invention du musée universitaire ». L'Ashmolean Museum présente, en effet, des innovations importantes par rapport aux anciens musées d'enseignement. Le musée est d'abord une véritable institution qui expose des collections de façon permanente et qui appelle à un plus large public que les seuls universitaires. Puis, le musée universitaire fait office d'instrument de travail ayant pour but de soutenir l'enseignement, au même titre que la bibliothèque et les archives.

Pour télescoper son histoire, l'âge d'or du musée universitaire se situe au XIX ${ }^{e}$ siècle. "In essence, the model adopted by (first generation) university museums during the golden age was that of the Ashmolean: the institutionalised integration of teaching, research and public display. " ${ }^{[2]}$ Le XX ${ }^{e}$ siècle marque, quant à lui, la prise de conscience par les universités « qu'elles [ont] accumulé des objets, des installations et des équipements d'enseignement de grande valeur historique ${ }^{[3]}$. 


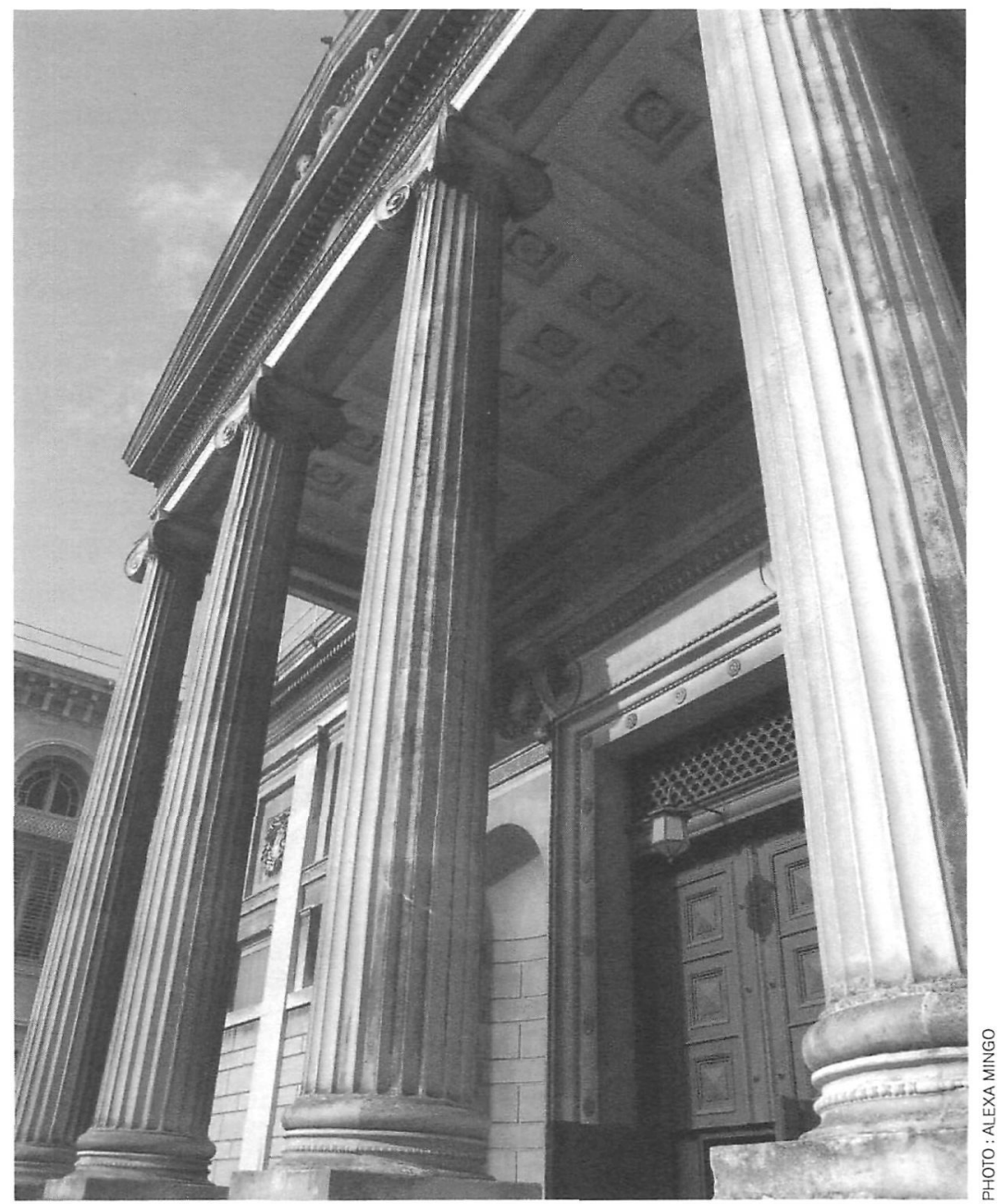

FIGURE 1

Entrée principale de l'Ashmolean Museum d'Oxford, Angleterre

Aujourd'hui, dans le monde, les musées et les collections universitaires sont aussi riches et importants que diversifiés. Cette diversité est l'une des raisons de la complexité de ce type de musée, puisqu'il existe aussi bien des musées universitaires d'histoire, de beaux-arts et d'ethnologie que d'archéologie, de médecine, de zoologie, de physique ou de géologie, sans compter les jardins botaniques, les observatoires astronomiques ou les musées d'art contemporain.

Les collections de ces musées ont souvent subi, au cours des dernières décennies, des modifications profondes quant à leur statut. Certains musées universitaires ont répondu favorablement à ces transformations et sont toujours dynamiques, alors que d'autres ont vécu ces changements avec difficulté. 
Ainsi, de nombreuses collections universitaires sont aujourd'hui dormantes. Étant donné leur caractère particulièrement pointu, elles sont souvent inconnues et inutilisées. Leur situation actuelle de dormance peut être la conséquence du changement de vocation de ces collections, qui ne sont plus que rarement utiles pour l'enseignement en raison des nouvelles méthodes d'instruction et de l'avènement de la technologie, ou de leur spécialisation qui fait en sorte qu'elles ne disposent que d'un faible auditoire, réel ou potentiel.

Cette réalité pousse à se questionner sur l'avenir de ces collections. Si rien n'est fait pour les sortir de l'oubli, qu'adviendra-t-il de ce patrimoine?

Une brève recension des modèles existants, soit diverses collections universitaires toujours en usage ou ayant été réhabilitées, permettra d'établir les options envisageables pour éviter la dormance de telles collections ou pour redynamiser les collections latentes. Les pistes proposées dans cette revue serviront de base au raisonnement développé dans notre proposition théorique. Bref, nous tenterons d'établir l'avenir qui peut être envisagé pour le patrimoine universitaire qui se trouve en dormance.

\section{Revue des modèles existants et leurs principes}

Une brève revue des modèles nous permettra d'étudier différents types de collections : le Classics Museum de la Victoria University of Wellington, les collections géologiques des universités hollandaises d'Amsterdam, de Delft, de Groningen et d'Utrecht, ainsi que le Jardin des Sciences de Strasbourg.

La collection d'objets antiques du Classics Museum de la Victoria University of Wellington (Nouvelle-Zélande) a été initiée à la fin des années 1940 par une professeure d'art grec, Denise Kalfas, qui a légué sa collection personnelle à l'université. Les pièces avaient été acquises dans le but de soutenir l'enseignement du département des «Classics».

La collection comprend de nombreuses poteries, principalement grecques, mais également minoennes, mycéniennes, chypriotes, étrusques et corinthiennes, des artefacts romains en verre, en bronze ou en marbre, des lampes, des pièces de monnaie, etc. En tout, la collection comprend 170 artefacts antiques et 135 pièces de monnaie.

Dès ses débuts, le rôle du musée de la Victoria University de Wellington a été de donner aux étudiants du département des "Classics » un accès direct à du matériel antique. Depuis, sa fonction première n'a pas changé : "The main role of the [...] Museum is to provide a home for a reasonably comprehensive 
coverage of the main forms, styles and materials of Greek, Roman, and Etruscan art, readily accessible to tutorials and students of Classics courses " ${ }^{[4]}$.

Tout d'abord, presque tous les objets de la collection sont en exposition de façon permanente dans le musée, donc à la vue de tous. Ensuite, le musée reste très lié à l'enseignement. De nombreux cours et séminaires sont donnés à même ses locaux et des visites de groupes scolaires ont lieu régulièrement. Les étudiants ont également un accès direct aux objets, qui sont tous identifiés par des cartels détaillés. Ils ont la possibilité d'examiner de près et de manipuler les artefacts, sous la supervision de la conservatrice. Enfin, une politique d'acquisition est établie et de nouvelles pièces sont régulièrement intégrées à la collection, grâce à des dons ou à des achats. Le musée est donc toujours en mouvement et de nombreuses personnes s'y intéressent, professeurs comme étudiants. Par ailleurs, le musée emploie une conservatrice et une administratrice, chacune cumulant des fonctions au programme d'études classiques et au Classics Museum.

Principe d'association - Le lien direct entre des programmes d'étude et un musée amène les étudiants à être actifs au sein du musée universitaire. Des acquisitions régulières montrent la vigueur de l'institution.

Les universités hollandaises d'Amsterdam, de Delft, de Groningen et d'Utrecht possédaient de vastes collections géologiques. L'enseignement et la recherche en géologie ayant grandement changé à partir des années 1960, chacune des universités se retrouvait avec un grand nombre de pièces géologiques, dont une grande partie était inutilisée. Grâce à une initiative nationale, la Netherlands Foundation of Academic Heritage, un inventaire exhaustif de certaines collections a pu être fait dans les cinq plus anciennes universités des Pays-Bas. Un des projets, réalisé à partir de l'an 2000, concernait justement les collections géologiques.

Acquises depuis le dernier quart du XIX ${ }^{e}$ siècle, les collections des universités d'Amsterdam, de Delft, de Groningen et d'Utrecht, qui comptaient environ deux millions de pièces géologiques, ont d'abord été divisées en 842 sous-collections. Une base de données ${ }^{[5]}$ de ces sous-groupes répertoriant, outre l'information générale sur le groupe, la qualité de la sous-collection et des possibilités pour un usage futur, a été établie.

Ces sous-collections ont ensuite été classées selon trois catégories, qui allaient déterminer leur avenir au sein de l'université :

1] les sous-collections toujours utilisées par les facultés pour l'enseignement et la recherche ;

2] les sous-collections inutilisées pour l'éducation et la recherche à l'intérieur de l'université, mais toujours considérées comme importantes sur le plan scientifique ou culturel ;
[4]

Victoria University of Wellington Classics Museum, Policy Framework.

[5]

La base de données était accessible sur le site Internet du Museum of the Technical de l'université de Delft. 
KELLY, Melanie (dir.). Managing University Museums. Paris : Organisation de coopération et de développement économiques (OCDE), 2001,

p. 98.
3] les sous-collections considérées comme ayant peu d'importance scientifique et/ou culturelle.

Après avoir obtenu l'accord de toutes les universités pour la cession de collections, l'opération visant à offrir les « unused collections to potential new users ${ }^{[6]}$ a pu être lancée. Toutes les sous-collections des catégories 2 et 3 ont été offertes aux universités, aux musées et aux commissions géologiques, autant aux Pays-Bas qu'ailleurs dans le monde, par le biais du site Internet du Museum of the Technical de l'université de Delft. On a ainsi pu réduire le volume des collections géologiques inutilisées des universités hollandaises d'environ $30 \%$. Par son entrée dans une nouvelle institution, chacune des sous-collections peut espérer une deuxième vie active et profitable.

Il s'agit d'une alternative délicate, puisqu'elle exige la cession de collections et que le sujet de l'aliénation d'objets est toujours épineux au sein des musées. Cependant, lorsqu'il est question de collections dormantes et inutilisées, la cession semble une solution envisageable.

Principe d'aliénation - La cession d'objets permet de sortir des collections universitaires de leur dormance grâce à leur entrée dans une nouvelle institution où elles amélioreront leur statut et deviendront utiles à la communauté dans laquelle elles viennent d'être incorporées.

FIGURE 2

Couverture du livre Le Jardin Botanique, Collection Images de I'Université Louis Pasteur, 2000.

\section{Le Jardin botanique}

Photographies Bernard Braesch
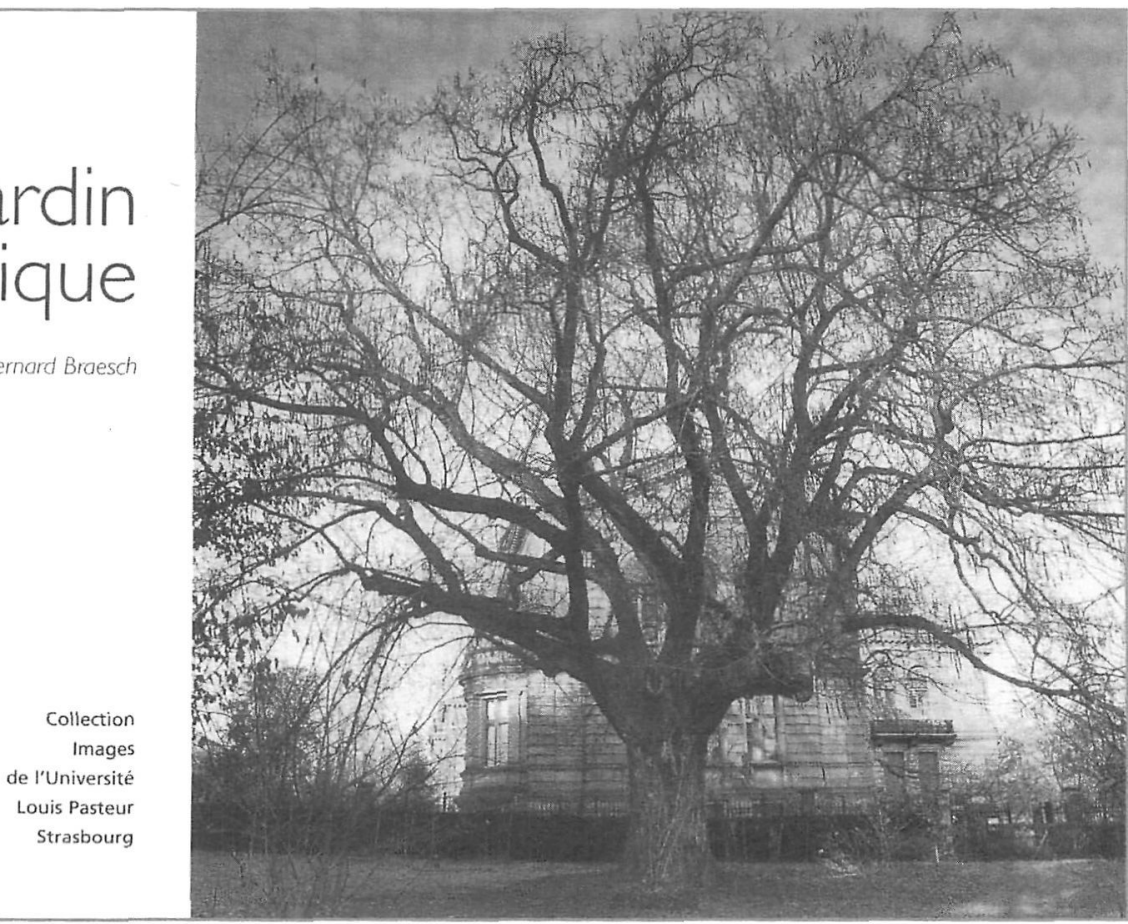
L'Université Louis Pasteur (ULP) à Strasbourg, qui se distingue sur le plan scientifique à l'échelle européenne, possède un large éventail de collections scientifiques. L'université, par le biais de la Mission culture scientifique et technique et grâce au soutien de la ville et de la Région Alsace, souhaite créer une entité intégrant toutes ses collections scientifiques : le Jardin des Sciences.

Le Jardin des Sciences veut en effet réunir les collections de minéralogie, de zoologie, de botanique, de paléontologie, d'instruments astronomiques, d'instruments de physique, de médecine, etc. Ce projet de Jardin des Sciences, qui veut "garantir la conservation et la valorisation du patrimoine scientifique de l'ULP " ${ }^{[1]}$, est vaste et ambitieux. Il prévoit entre autres un planétarium, un observatoire, un jardin botanique, un musée de sismologie, un musée des sciences, une station minéralogique et un institut de physique.

À l'heure actuelle, certaines actions sont posées afin d'engager la sauvegarde des collections, d'inventorier les objets et de créer des expositions à caractère scientifique. Tout d'abord, on a effectué l'inventaire des instruments astronomiques, conduisant à la création d'une base de données pour les 200 objets de l'observatoire astronomique, processus qui doit par la suite être appliqué aux autres collections d'instruments scientifiques (sismologie, magnétisme, minéralogie). Ensuite, des étudiants ont créé un site Internet, sous la supervision de la Mission culture scientifique et technique, afin d'y présenter l'ensemble des collections et des musées des universités de Strasbourg. Des expositions à caractère scientifique sont par ailleurs organisées, en préfiguration de celles qui auront lieu dans les divers secteurs du Jardin des Sciences.

L'objectif du Jardin des Sciences de Strasbourg « est de créer un grand équipement de culture scientifique reposant sur les différentes structures muséales et collections, en les associant aux multiples actions de médiation de culture scientifique et technique engagées au sein de l'université " ${ }^{[8]}$, dans le but de développer différents espaces qui serviront tant à la visite et à la promenade qu'aux débats ou aux rencontres sur les diverses représentations de la science. Sans perdre l'idée qu'il s'agit de collections universitaires, le Jardin des Sciences se veut ouvert au grand public en prenant la forme d'un parcours muséal, tout en servant d'outil à la formation et à la recherche des universitaires de Strasbourg et du monde.

Principe d'intégration - La réunion de plusieurs collections spécialisées en une même entité unifiée assure la sauvegarde des collections, tout en permettant une meilleure gestion et de nouvelles possibilités, comme des expositions, la création de bases de données documentaires et un lien direct avec les recherches universitaires actuelles.

\section{[7]}

Université Louis Pasteur Strasbourg I. Jardin des Sciences. <www-ulp.u-strasbg. fr/article.php/0/5/1-078325-420/jardin-dessciences\#i_166706416_81> (consulté le 19 février 2007). [8]

SOUBIRAN, Sébastien. "Patrimoine des universités et médiation scientifique . La Lettre de l'OCIM (Office de Coopération et d'Information Muséographiques), $\mathrm{n}^{\circ}$ 109, janvier-février 2007, p. 39. 


\section{Renaissance des collections universitaires dormantes}

Plusieurs collections universitaires se trouvent actuellement dans un état de dormance et ce, pour diverses raisons : manque de ressources humaines qualifiées et intéressées, manque de ressources financières, besoin d'espace ou de locaux dans l'université qui repousse les collections dans les greniers ou les sous-sols, manque d'intérêt de la communauté universitaire, perte de leur fonction de recherche ou d'enseignement, etc. D'ailleurs, dès que la mission originale de la collection " disparaît et que, dans le contexte universitaire, elle [la collection] ne sert plus que d'objet d'exposition, elle risque d'être totalement abandonnée " ${ }^{[9]}$. Tout cela concourt à ce que de nombreuses collections ne soient plus utilisées par la communautéuniversitaire et soient reléguées aux oubliettes. C'est le cas des différentes collections del'Université Laval ${ }^{[10]}$, notamment depuis la fermeture officielle du Centre muséographique en 2000, ou encore de celles du Musée d'ethnographie de l'Université Bordeaux II. de savoirs et d'expériences". Louvain, $n^{\circ} 156$, juin 2005

Pour un total de près de un million de pièces.

[11]

CLERQ, Steven W.G de. "What if We Weren't Here?" Museologia, no 3, 2003,

p. 150.

La recension de quelques collections universitaires nous a permis d'établir que des principes existent pour garder vivantes ces collections. Nous élaborons ici une proposition théorique afin d'appuyer le réveil des collections dormantes, et ce, en développant d'abord deux voies conceptuelles : l'alliance stratégique et l'autosuffisance.

\section{L'alliance stratégique}

a) L'objet comme document : inclusion des collections universitaires au lot documentaire de l'université

Pour redonner sens à des collections universitaires, il peut être intéressant de songer à une union, organisationnelle et virtuelle, avec l'ensemble documentaire del'université, c'est-à-dire avec les bibliothèques et les archives. Bien que chacun des documents en question (objet, livre, document d'archive, œuvre d'art, film, disque, etc.) ait ses propres spécificités et finalités, le but est commun : documenter et supporter l'étude. Cette mission de préservation, commune aux musées et aux bibliothèques, justifie un certain rapprochement de la part de ces deux entités. Évidemment, il est très difficile, voire impossible, de réunir physiquement tous ces documents. C'est pourquoi une alliance virtuelle et organisationnelle doit être privilégiée.

"Together with libraries and archives, we [university museums] have to develop an interdisciplinary approach for the definition enhancement and access of our scientific heritage. " "11] Cette réunion dans une même unité virtuelle permettrait aux collections dormantes, peu connues ou inutilisées, de reprendre vie grâce à un repérage plus facile des objets. 
Cela pourrait commencer par le rassemblement des secteurs concernés au sein d'une même unité administrative, dont la direction commune a une mission de documentation et de sauvegarde. On peut donc penser que le même support, financier, matériel et humain, sera apporté à tous les outils de recherche universitaire : bibliothèques, archives et collections. Par exemple, cela a déjà été fait en Grande-Bretagne, à l'université d'Aberdeen, où le musée Marischal, un musée d'anthropologie et d'ethnologie, a été placé sous le "Directorat des systèmes d'information ", avec les bibliothèques et les archives.

Ensuite, si les moyens nécessaires existent, la numérisation des objets des collections est une possibilité très intéressante. Cette numérisation, ainsi que celle des documents d'archives par exemple, permettrait d'inclure tous les documents (livres, objets et documents d'archives) dans une même base de données gérée par un système intranet. Les recherches faites par les étudiants ou les professeurs seraient alors grandement étendues. Pour une recherche ciblée, par sujet par exemple, la base de données répondrait en indiquant les livres, bien sûr, mais également les objets présents dans les collections qui se rapportent à ce thème. C'est d'ailleurs le système qui est utilisé pour les diapositives à la Diapothèque à l'Université Laval, un secteur de la bibliothèque des sciences humaines et sociales qui est intégré au catalogue Ariane de consultation du fonds en bibliothèque.

Évidemment, cette avenue nécessite des changements fondamentaux au sein des institutions - musées universitaires comme universités et l'allocation de budgets pour permettre une telle intégration.

Grâce à cette alternative, les collections pourraient sortir de leur dormance en étant à portée de main des étudiants et des chercheurs. Ces derniers sauraient enfin ce que possède l'université en termes de collections et de "documents-objets". On peut penser que leur intérêt pour ce patrimoine ne pourrait qu'en être accru.

\section{b) Regroupements de musées universitaires et de sites Web}

Le Web et son ouverture sur le monde permettent nombre de nouvelles possibilités pour les musées et les collections universitaires.

On peut d'abord évoquer tous les regroupements de musées et les collections universitaires qui ont une vitrine sur le Web. En 1987, le premier de ces regroupements, le University Museums Group (UMG), est formé en GrandeBretagne. Ses rôles principaux sont de promouvoir les intérêts des musées universitaires, de créer un forum pour les rencontres intermusées et d'agir comme groupe de pression pour représenter les musées universitaires. 
En 2000, plusieurs pays européens s'intéressent à la question, ce qui mène à la création du réseau Universeum, à la suite de la signature de la Déclaration de Halle par une douzaine de représentants de musées universitaires européens. Leur but premier est de " partager des connaissances et expériences [...] et d'entreprendre dés projets communs visant à améliorer l'accès aux collections à tous les niveaux ${ }^{\left[{ }^{[12]}\right.}$.

Enfin, en 2001, l'attention pour les musées universitaires conduit à un regroupement international. L'UMAC (University Museums and Collections), un des comités de l'ICOM, (International Council of Museums) est créé et veut lui aussi veiller aux intérêts des musées universitaires. L'objectif de ce comité est de protéger le patrimoine qui est sous la responsabilité des universités et de permettre ainsi à ses membres d'échanger savoirs et connaissances.

Des sites Internet peuvent également regrouper divers musées et collections sous une même thématique. Par exemple, le Global Egyptian

La Smithsonian Institution n'est pas une université à proprement parler, mais, comme il s'agit d'un institut de recherche et d'enseignement, nous pouvons considérer ses collections au même titre que toute collection universitaire. Museum (GEM) réunit des collections égyptiennes dans le monde, dans le but d'en accroître la diffusion. Il ne s'agit pas uniquement de collections universitaires, puisque toutes, publiques comme privées, sont appelées à faire partie du GEM. On y trouve, par exemple, les collections de l'Institut de papyrologie et d'égyptologie de l'Université de Lille III, mais également celles du Rijksmuseum de Leiden aux Pays-Bas.

\section{L'autosuffisance}

Un des points essentiels de l'autosuffisance des collections universitaires est vraisemblablement la recherche. Cette recherche permet d'ailleurs la réactualisation des collections en dormance. Une collection sur laquelle on effectue de la recherche ne peut être dormante, parce qu'elle est appréciée, étudiée et comprise. Une collection étudiée est donc jusqu'à un certain point une collection autosuffisante.

C'est pourquoi la recherche est un aspect non négligeable de la réhabilitation des collections dormantes. L'intérêt porté à une collection par le biais de la recherche lui permet de demeurer vivace et de se développer par la qualité et la quantité des connaissances détenues à son sujet.

De nombreuses collections universitaires ont été réactualisées par la recherche. Dans certains cas, cette recherche a même amené des percées scientifiques majeures. La collection universitaire dormante qui a été réétudiée et qui a fait couler le plus d'encre est sans doute la collection du schiste de Burgess de Charles Doolittle Walcott, conservée à la Smithsonian Institution de Washington ${ }^{[13]}$. Walcott a fait la découverte des premiers fossiles du schiste de Burgess en 1909. Jusqu'en 1917, il a retiré de cette carrière canadienne 65000 
fossiles de plantes et d'animaux. Il a étudié cette collection jusqu'à sa mort en 1927, puis tout a été entreposé à la Smithsonian Institution jusqu'à ce que de nouveaux chercheurs s'intéressent à la question, à la fin des années 1960. Un professeur de l'université de Cambridge, Harry Whittington, et deux étudiants, Simon Conway-Morris et Derek Briggs, étudient alors et réexaminent les fossiles ainsi que le travail de Walcott, de façon très détaillée, ce qui mène à la réinterprétation du schiste de Burgess. Cette découverte est le résultat de recherches effectuées sur des collections spécialisées en dormance depuis de très nombreuses années.

Ce cas, bien que hors du commun, permet de voir à quel point la recherche est essentielle dans le processus de réhabilitation de collections dormantes. Plus une collection est étudiée, mieux elle peut être connue et reconnue; mieux elle est étudiée, plus elle devient autosuffisante.

\section{Proposition pour le réveil des collections en dormance :}

\section{la Réserve ouverte et virtuelle des universités (ROVU)}

L'alliance stratégique des collections et leur autosuffisance semblent être, lorsque prises au pied de la lettre, deux concepts antinomiques, qui peuvent par ailleurs être complémentaires quand on traite des collections universitaires. Puisque ces deux voies semblent apporter des propositions appropriées au problème des collections universitaires dormantes, ne vaudrait-il pas mieux tenter de les combiner?

Les pistes étudiées précédemment nous permettent maintenant de construire une proposition cohérente pour inciter au réveil des collections universitaires en dormance.

En cette ère du Web et du multimédia, notre proposition, pour être cohérente, ne peut avoir qu'une forme majoritairement virtuelle. Cependant, nous croyons que nous ne pouvons laisser tomber complètement l'aspect matériel des collections universitaires, car «la nécessité d'une pédagogie par l'objet est réaffirmée [et que, dans] notre nouveau monde du virtuel [...], le musée est l'un des endroits où persiste un rapport au réel, à l'objet " "

Il est d'abord important de noter qu'un des projets actuellement mis en place et qui a retenu notre attention pour la définition de notre proposition est le Reciprocal Research Network (RRN) du Museum of Anthropology de la University of British Columbia. Ce projet de grande envergure, supporté par la Fondation canadienne de l'innovation, présente des aspects très intéressants sur les plans de la mise en réseau et de la virtualité des collections universitaires, certains éléments pouvant être appliqués à plus petite échelle.
FERRIOT, Dominique.

"Musées des sciences et universités». Revue culturelle de l'Université de Bourgogne, p. 2. 
Le cas de l'Australian University Museums on Line (AUMOL), répertoire électronique des collections universitaires australiennes, est également intéressant pour la notion de réseau virtuel.

Notre proposition pour raviver les collections dormantes est l'instauration d'un musée / réserve, virtuel et ouvert, rassemblant les diverses collections spécialisées que l'université possède : la Réserve ouverte et virtuelle des Universités (ROVU).

Tout d'abord, d'un point de vue administratif, il importe que les collections universitaires soient gérées par le secteur approprié. Il convient donc de privilégier la section «bibliothèques et archives " plutôt qu'un quelconque département, même si ce dernier est concerné par la ou les collections. Ainsi, les missions de préservation et de documentation seront au cœur de la gestion des collections.

Ensuite, point majeur de notre proposition, le musée se doit d'être une réserve ouverte, une bibliothèque d'objets, afin d'être accessible à toute la communauté universitaire, au même titre que la bibliothèque ou les archives. Il est primordial que tout étudiant ou tout professeur qui souhaite voir ou étudier un objet puisse le faire avec toutes les précautions nécessaires et en observant, bien évidemment, les règles primaires de conservation préventive. Cet accès facilité aux collections encouragerait, il va sans dire, les étudiants à effectuer de la recherche sur divers aspects des collections, en culture matérielle notamment, et ce dans toutes les disciplines représentées au musée.

Afin d'être aussi accessible et ouvert aux différents chercheurs qu'un centre d'archives, le musée se doit de posséder une base de données intelligible, complète et détaillée, ce qui nécessite un moteur de recherche efficace, des fiches d'objets normalisées et des représentations numérisées précises de chaque objet. De plus, chacune des fiches doit comprendre toutes les références qui existent au sujet de l'objet : publications diverses, recherches, liens vers d'autres objets associés, ainsi que tout document audio ou vidéo approprié.

Cette base de données doit être accessible à partir de postes informatiques situés à même le musée / réserve, mais également par le biais d'Internet. Dans le but de permettre une recherche rapide et pour faciliter la protection des droits de propriété intellectuelle des images, les internautes auraient alors la possibilité de consulter seulement la version abrégée des fiches d'objets.

L'autre point majeur de notre proposition est la mise en réseau du musée, grâce au Web, avec d'autres musées universitaires qui ont des intérêts communs. C'est pourquoi nous parlons de musée / réserve virtuel. Cette intégration permettrait, dans un premier temps, de s'assurer d'une entraide sur les plans 
scientifique, organisationnel et muséologique, grâce aux liens qui unissent les diverses institutions. L'objectif poursuivi par cette association est de permettre aux musées universitaires dont les collections sont peu connues ou sousutilisées, voire dormantes, de se faire connaître autant dans leur propre université que dans les autres institutions.

Pour réaliser cet objectif, chacun des musées doit permettre l'accès à sa base de données détaillée. Les objets numérisés des autres collections universitaires seraient donc inclus virtuellement dans la base de données de notre ROVU, qui constituerait alors une réserve virtuelle élargie. Il serait alors possible d'englober, dans une seule recherche, les collections de plusieurs universités.

Chacune des collections universitaires du réseau devient ainsi une référence à distance, ce qui permet notamment queles collections del'université soient utilisées pour des projets de recherche d'autres universités.

Le musée universitaire / réserve ouverte permet d'abord de faire revivre des collections dormantes en donnant un accès privilégié aux objets pour les chercheurs, les étudiants et les professeurs. L'association de réserves virtuelles donne aux collections une visibilité internationale par le biais du réseau et du Web, encourageant le dynamisme des musées universitaires au sein des universités, mais elle peut également permettre d'intéresser un public élargi.

\section{Avenir des musées et des collections universitaires}

En cette première décennie du XXI ${ }^{e}$ siècle, nous pouvons observer des changements fondamentaux dans le monde muséal, spécialement dans le rôle des musées ${ }^{[15]}$. En étant de " plus en plus exposés à des critères chiffrés et économiques, les musées risquent de ne plus exercer leur activité principale, l'exposition de collections " ${ }^{[16]}$. Pour tous les musées, mais particulièrement pour les musées universitaires, nous pouvons dire que les collections sont extrêmement importantes. L'enjeu principal des musées devient le nombre de visiteurs et la quantité d'expositions temporaires présentant des chefsd'œuvre reconnus, l'attrait pour les collections permanentes diminuant sans cesse.

Ce rôle des musées qui change si fondamentalement touche d'une double façon les musées universitaires, puisqu'ils se trouvent à la confluence de deux mondes qui bougent rapidement en cette période de grande mouvance sociétale : le monde muséal et le monde universitaire.

\section{[15]}

Voir à ce sujet : MAIRESSE, François et André DESVALLÉES. Vers une redéfinition du musée. Paris : L'Harmattan, 2007, 228 p.

[16]

ICOM Autriche.

Musées et patrimoine universel. $<$ http://www.icom-oesterreich. at/2007/index.html $>$ (consulté le 9 avril 2007). 
ICOM. UMAC. $<$ http://

publicus.culture.hu-berlin.de/ umac/2007/> (consulté le 26 février 2007)
C'est pourquoi les musées universitaires doivent redéfinir leur rôle, au sein de la communauté universitaire et au sein même de la société. Ils doivent se tailler une place de choix dans ces deux mondes et établir quelles sont leurs priorités, en prenant en compte les réalités muséales et universitaires. "This implies not only a re-valuation of [the] three fundamental missions: research, teaching and public display, but also the question how that affects our heritage, both tangible and intangible. " ${ }^{[17]}$ Cette question sera d'ailleurs au cœur des discussions de la prochaine rencontre annuelle de l'UMAC, lors de la XXI Conférence générale de l'ICOM qui se tiendra à Vienne, en Autriche, du 19 au 24 août 2007.

Puisque les universités elles-mêmes vivent une crise identitaire, notamment avec les coupures budgétaires et l'implication de l'entreprise privée dans l'enseignement supérieur, nous pouvons dès lors nous questionner sur ce qu'elles prévoiront pour leurs musées et attendront d'eux. Il importe donc de s'attarder plus avant à ces changements cruciaux et d'entreprendre une réflexion collective au sujet de l'avenir des musées et des collections au sein des universités. Cette réflexion pourrait par exemple être pilotée, dans le cas du Canada, par l'AMC (Association des musées canadiens), la SMQ(Société des musées québécois) et l'Association des universités et collèges du Canada, afin d'envisager de concert l'avenir du patrimoine universitaire de façon renouvelée. 
The implementation of new teaching methods and technologies has caused a breakdown in the world of university collections. Consequently, those collections seem to have an increasingly less important role to play. With the added difficulties concerning financial, human, and material resources, it becomes evident why many university collections have been sidelined and are now dormant. If nothing is done to change the disinterest in such collections and to stop their decline, the future of that major cultural patrimony is questionable.

However, several university collections have succeeded in remaining active or have been restored, proving that options do exist for the revival of dormant university collections. Currently, the utility of the virtual option is clearly indispensable; however, it does not mean that the material aspect of objects in thee collections should be ignored. For that reason, we have conceived the idea of a university museum which would act as an open and virtual reserve: the ROVU, Réserve ouverte et virtuelle des universités (Open and Virtual Museum Reserve). The ROVU would include the little-used or -publicized special collections of certain universities, allowing the collections to become active once again, generating new interest and facilitating student researcher and visitor access.

Given the major developments that have occurred concerning the perceived role of museums, the future of university museums is a crucial concern. The university museum community must reflect upon the situation.

In order to properly understand the issue, we will first present a brief summary of the history of university collections, beginning from the opening of the Ashmolean Museum at Oxford in 1683 and continuing up to the present day.

We will then review several existing models by examining examples of the diverse university collections that are still in use or have been restored, including the Classics Museum at the Victoria University of Wellington (New Zealand), the geological collections of the Dutch universities in Amsterdam, Delft, Groningen and Utrecht, and the Jardin des Sciences in Strasbourg (France). Those examples 
will serve to demonstrate several possibilities for avoiding collection dormancy or revitalizing latent collections. The study of those collections will evoke certain principles and allow us to identify conditions that are favourable to the survival of university collections, such as a direct link between study programs and the museum, an online presentation of objects via the Internet, the cessation of objects, the integration of several specialized collections within the same unified entity, etc.

Next, to lend our support to the revival of dormant collections, we will introduce a theoretical proposition beginning with a description of the paths that lead to revival. There are two conceptual routes open to museums or university collections: integration or autonomy. We will see that each offers practical possibilities (integration of university collections in the documentation centres of the university, grouping university museums and their websites, and research), all of which will lead us to our proposed solution: the Réserve ouverte et virtuelle des universités.

The creation of an open and virtual museum/reserve, which would include diverse university collections, would open many doors for university patrimony. The ROVU has been conceived to operate on two levels. On the university level, it would act as an open reserve, a library of objects easily consulted by all student researchers. On the network level, it would operate as a virtual reserve, integrating the collections of each member university. Each university collection belonging to the network would become a reference available remotely, therefore increasing its visibility and scope.

That theoretical solution to the problem of dormant university collections is one possible option if we are to prevent our university patrimony being forgotten. Although it is neither applicable universally as it is now, nor in the short term, it does incite discussion and new ideas, encouraging universities to reflect upon the future of their collections. 